\title{
Improved Ant Colony-Genetic Algorithm for Information Transmission Path Optimization in Remanufacturing Service System
}

Lei Wang ${ }^{1,2}$, Xu-Hui Xia ${ }^{1 *}$, Jian-Hua Cao ${ }^{1,2}$, Xiang Liu² and Jun-Wei Liu

\begin{abstract}
The information transmission path optimization (ITPO) can often affect the efficiency and accuracy of remanufacturing service. However, there is a greater degree of uncertainty and complexity in information transmission of remanufacturing service system, which leads to a critical need for designing planning models to deal with this added uncertainty and complexity. In this paper, a three-dimensional (3D) model of remanufacturing service information network for information transmission is developed, which combines the physic coordinate and the transmitted properties of all the devices in the remanufacturing service system. In order to solve the basic ITPO in the 3D model, an improved 3D ant colony algorithm (Improved AC) was put forward. Moreover, to further improve the operation efficiency of the algorithm, an improved ant colony-genetic algorithm (AC-GA) that combines the improved AC and genetic algorithm was developed. In addition, by taking the transmission of remanufacturing service demand information of certain roller as example, the effectiveness of AC-GA algorithm was analyzed and compared with that of improved AC, and the results demonstrated that AC-GA algorithm was superior to $A C$ algorithm in aspects of information transmission delay, information transmission cost, and rate of information loss.
\end{abstract}

Keywords: Remanufacturing service, Information transmission, Path optimization, Ant colony algorithm, Genetic algorithm

\section{Introduction}

As a key line in reversed supply chain, remanufacturing is an important approach to realize the recycling of retired products and economy sustainable development [1]. Since Lund proposed remanufacturing concept [2], researchers from home and abroad have conducted many researches on remanufacturing technology $[3,4]$, remanufacturing planning and scheduling $[5,6]$, remanufacturing evaluation and decision [7] and achieved substantial findings. Remanufacturing service (RMS) [8] is a web-based remanufacturing and service model based on information technology and cloud manufacturing technology [9]. Remanufacturing service process involves various types of massive data including demand

\footnotetext{
*Correspondence: xiaxuhui@wust.edu.cn

${ }^{1}$ Hubei Key Laboratory of Mechanical Transmission and Manufacturing Engineering, Wuhan University of Science and Technology,

Wuhan 430081, China

Full list of author information is available at the end of the article
}

information, manufacturing and processing information, logistics information. The main information flow is shown in Figure 1.

As remanufacturing service process is a highly customized process involving information in various types and large amount, a series of problems will be generated in construction of information platform and information service process. For example, there is significant difference in type, usage degree, and remanufacturability of waste products, which increases the difficulty degree in information preprocessing, waste product resource classification and remanufacturable product information collection. The customer demand of remanufacturing service is of diversity and randomness, and the incoming time and initial status of remanufacturable products are uncertain. All these factors lead to the challenge to demand information matching, process information matching, and decision information processing mechanism; Since remanufacturing 


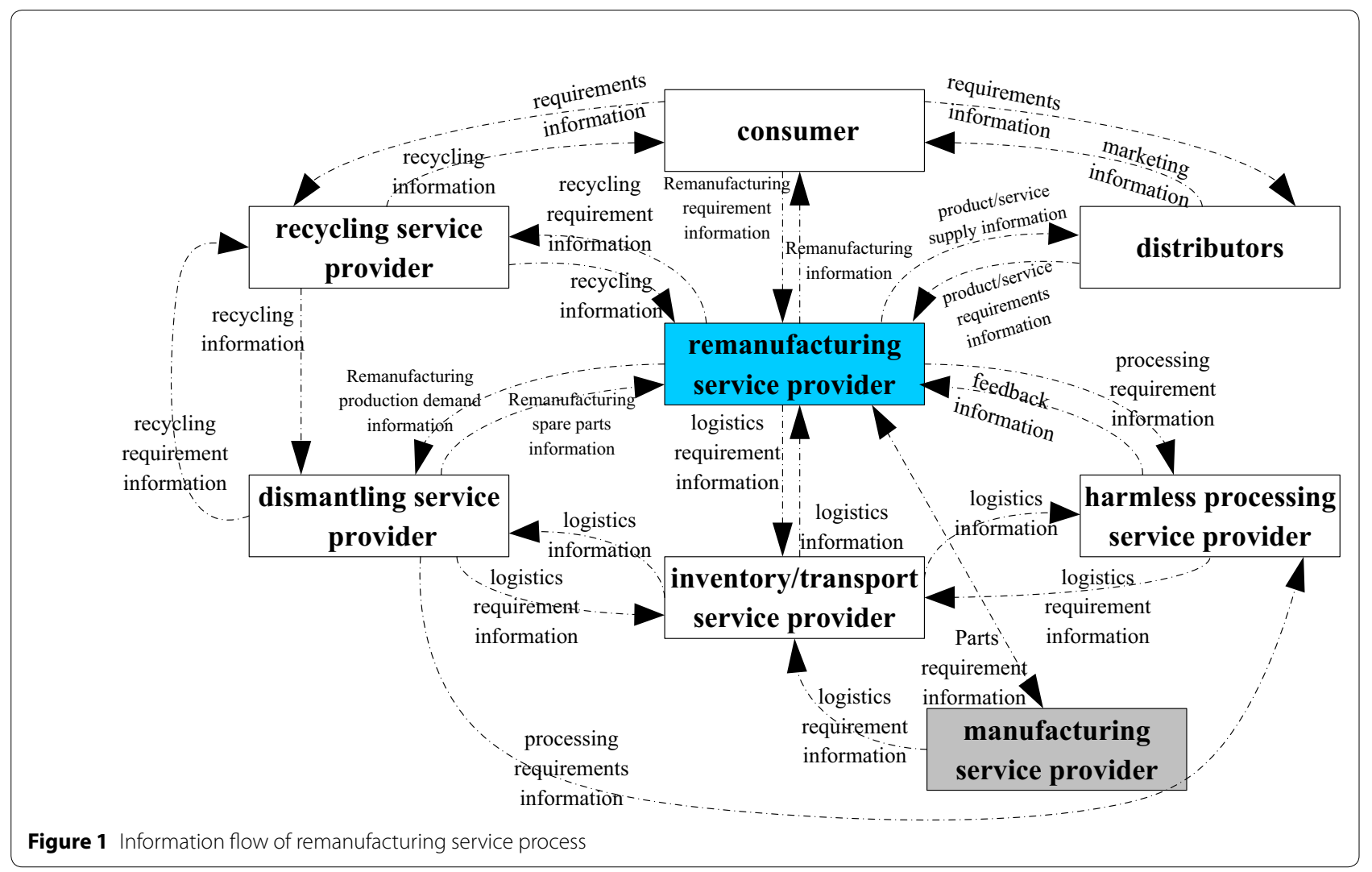

service system enjoys a cross-enterprise and crossregion information network, seeking proper information transmission nodes and paths in such network and realizing information source cross transmission to secure the real-time and accurate transmission of information resource are issues to be addressed for the establishment of informatizational service platform of remanufacturing service system.

Regarding above problems, researchers have conducted large amounts of researches on information collection technology, information data structure processing technology, and information matching technology $[10,11]$, and have built information service modes that suitable for various areas [12]. However, in the aspect of information transmission, researches are insufficient. As we know that an accurate and reliable information transmission plays a vital role in realizing the information service of remanufacturing service system [13], if information network fails to secure the integrity in transmission and exchanging of collected and matched information, will not the information service quality be secured. On this basis, this paper proposed an optimization method of 3D information transmission path based on improved ant colony-genetic algorithm (AC-GA), moreover the effectiveness of such method was analyzed based on the case study of the remanufacturing service demand information transmission of a certain roller.

\section{Related Works}

Information path planning thought is originated with path planning problem. Path planning technology has long been used in fields such as obstacle avoiding and penetration flight of aircraft [14], GIS-based path planning [15], robot path planning [16], and communication route optimization [17]. The core of route planning is the design of route planning algorithm [18], which currently can be categorized into three types, such as traditional algorithm, graphics algorithm, Bio-inspired intelligent algorithm [17-19]. The advantages and disadvantages of these algorithms are shown in Table 1.

According to the characteristics of different information route planning algorithms, ant colony algorithm is regarded of significant advantage in remanufacturing service system information route optimization, because it has large amount of information resource involved in remanufacturing service system, numerous information transmission network nodes, and 3D distribution space. Being often introduced in 3D route planning problem of robot under complex environment [20], ant colony algorithm can divide the space between robot location 


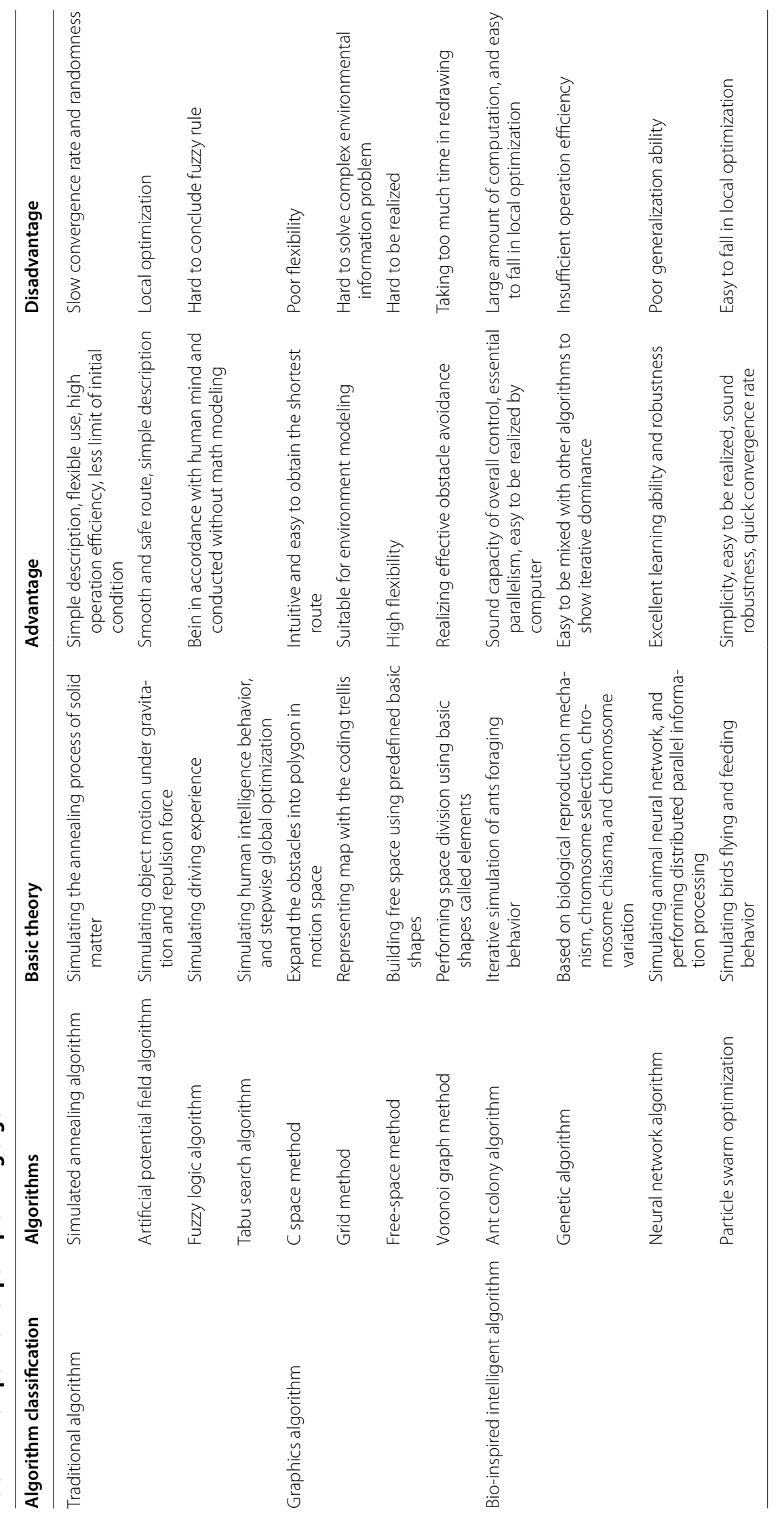


(original point) and destination point into three-dimensional grids and calculate the shortest optimized route between original point and destination point [21]. With strong robustness, ant colony algorithm can realize parallel calculation and is easy to be combined with other algorithms, which is more suitable for solving large-scale dynamic optimization problem [22, 23]. Some researchers have introduced ant colony algorithm in functional optimization and solution of continuous space and fast optimization problem of proliferative process route [24, 25]. The application of ant colony algorithm in self-organized service recommendation network further proved that such algorithm can effectively improve the success rate and recall ratio of service discovery [26]. However due to the large computation amount of ant colony algorithm, this paper proposed an improved ant colony algorithm (Improved AC) by combining normal ant colony algorithm with genetic algorithm than features sound convergence, less computation time, higher robustness, and iterative advantages, and applied the improved AC to solve information transmission route optimization problem of remanufacturing service system.

\section{Framework of Information Transmission Network}

RMS includes both remanufacturing servitization and productive service. Remanufacturing servitization provides overall solution of remanufacturing engineering and remanufacturing products for customers. Productive service provides professional services for remanufacturing enterprises, such as disassembling of machinery, cleaning and detection of parts, remanufacturing processing, assembling, running and information-based remanufacturing [8]. RMS involves resource, organization, service value during interaction process [27]. To describe the coordinates of the information conversion devices, a three-dimensional (3D) model of remanufacturing service information network for information transmission was developed. This model combines the physic coordinate and the transmitted properties of all the devices in the network, as shown in Figure 2.

In Figure 2, $X$ axis, $Y$ axis and $Z$ axis is the physic coordinate of the network $N(V, E)$. In $N(V, E), V$ is the collection of all network nodes (exchangers, routers and hosts), $E$ is the collection of all edges in the figure, where in each edge represents a direct communication path between two adjacent points. Each routing node in information transmission routing network $N(V, E)$ is corresponded to an only routing node coordinate $p(u, \omega, i)$. Each $p(u, \omega, i) \in V$ has three attributes: including information transmission delay $(p)$, information communication $\operatorname{cost}(p)$ and information packet_loss $(p)$, the attribute parameter can be represented by $<\operatorname{delay}(p), \operatorname{cost}(p)$, packet_loss $(p)>$. Moreover, the attribute parameter of each edge $e \in E$ can be represented by $<$ delay $(e), \operatorname{cost}(e)$, packet_loss $(e)>$. The 3D map of routing nodes that corresponds with remanufacturing service information transmission network $N(V, E)$ in Figure 2 is shown in Figure 3.

\section{Problem Statement}

High-efficiency space routing planning is the guarantee for space information transmission [28]. Remanufacturing service information transmission routing planning is to seek an optimal remanufacturing service information communication path from route starting point $S \in V$ to target routing point $M \in\{V-\{s\}\}$ in the $3 \mathrm{D}$ information transmission routing network $N(V, E)$, as shown in Figure 3, where the disabled nodes (information transmission nodes in Load/ fault) are distributed. The communication service is guaranteed to meet constraint conditions, such as information delay, commutation cost, information packet_loss.

Suppose the network $N(V, E)$ is symmetrical and there is only one edge between two adjacent joints. During information communication $T(S, M)$ process from original point $S$ to target point $M$, there are 3 attributes including information transmission delay $(T(S, M))$, information communication cost $(T(S, M))$, and information packet_loss $(T(S, M))$ :

$$
\begin{aligned}
& \operatorname{delay}(T(S, M))=\sum_{i}^{n_{s}} t_{\mathrm{d}}^{i} / n_{\mathrm{s}}, \\
& \operatorname{cost}(T(S, M))=\sum_{i}^{N} e_{i}, \\
& \operatorname{packet\_ loss}(T(S, M))=1-D_{s} / \sum_{i=1}^{N} D_{i},
\end{aligned}
$$

where delay $(T(S, M))$ is the information transmission delay, i.e., the mean transit time from information original point to information receiving user $(\mu \mathrm{s}) . n_{\mathrm{s}}$ is the number of originating node. $t_{\mathrm{d}}^{i}$ is the time interval from sending data at originating node i to information receiving user $(\mu \mathrm{s})$. cost $(T(S, M))$ is information communication cost, i.e., the total unit energy consumption for information communication transmission. $e_{i}$ is the unit energy consumed by routing node $i$ in receiving and sending data. $N$ is the number of all routing nodes that are involved in $T(S, M)$ transmission path. packet $\operatorname{loss}(T(S, M))$ is the probability that information fails to be transferred to user nodes under the condition where the average load factor of the route $k=\sum_{i=1}^{N} k_{i} / N . k_{i}$ is the load factor of the $i$ th routing node in the information 


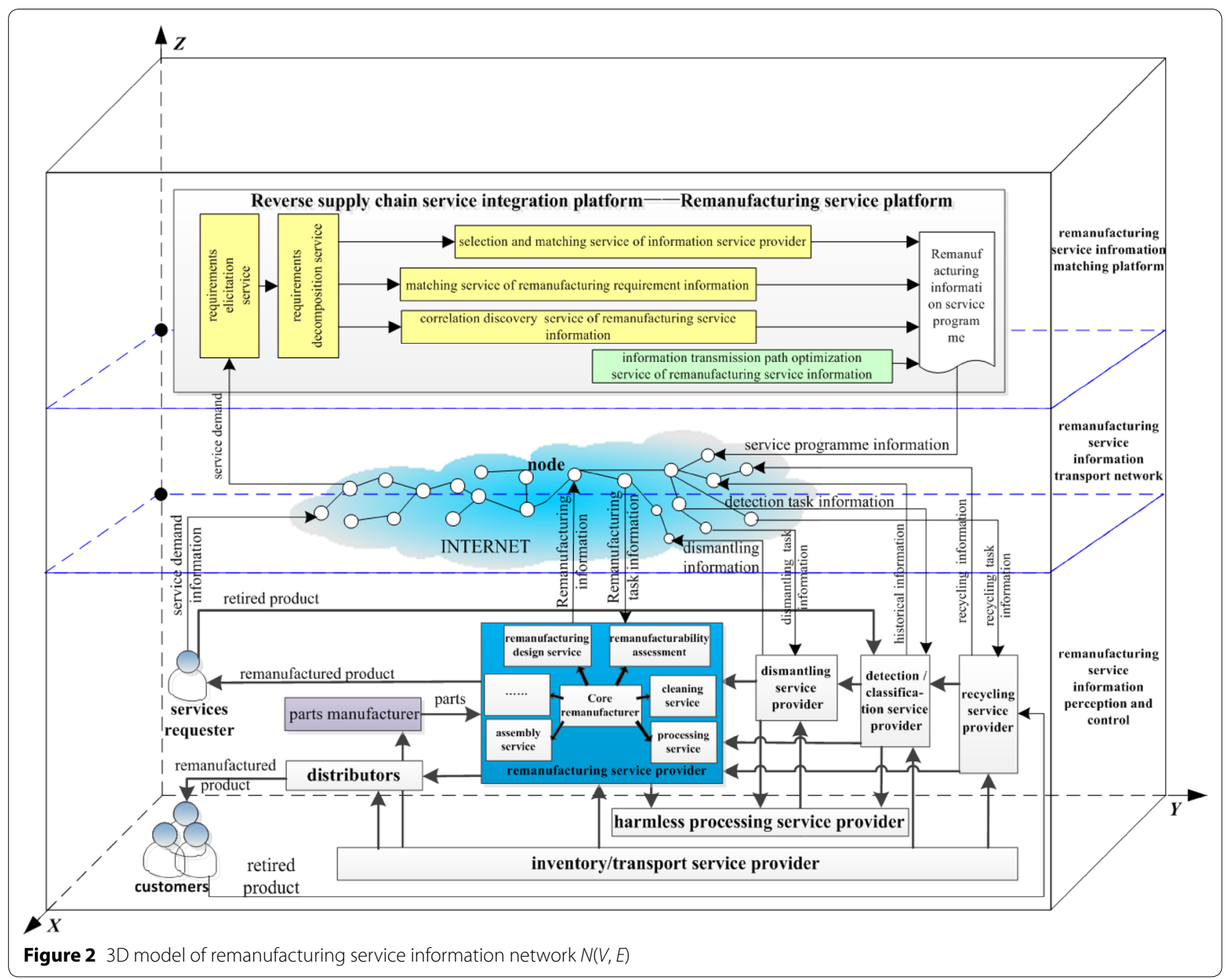

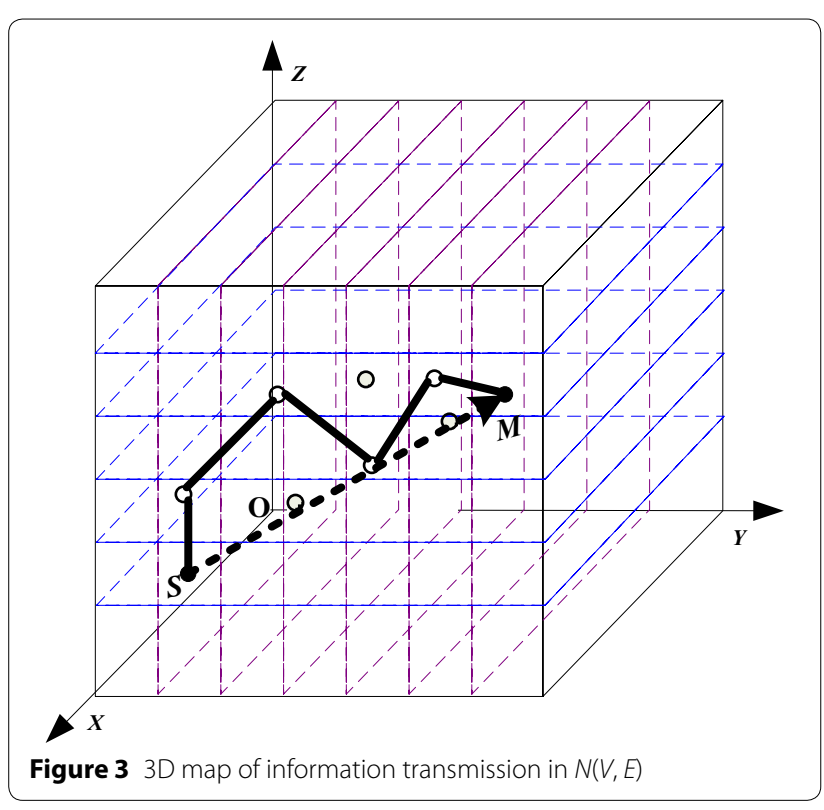

transmission route. $\sum_{i=1}^{N} D_{i}$ is the total amount of data sent from all routing nodes in $T(S, M)$ transmission path, $D_{i}$ is the amount of data sent from the node $i . D_{\mathrm{s}}$ is the total amount of data received by user node $M$.

\section{Optimization Method for Information Transmission Path}

\subsection{The Improved Ant Colony Algorithm (Improved AC) for Path Planning}

Basic ant colony algorithms are only suitable for 2D path planning problem [26]. However, regarding the 3D path planning problem in 3D map of information transmission routing network $N(V, E)$ shown in Figure 3, this paper proposes a 3D path optimization AC algorithm for remanufacturing service information transmission by referring the thought of 3D space robot path planning ant colony algorithm, and concrete steps are shown below.

Step 1: Coordinate transformation. As shown in Figure 3 , the straight-line distance from information original 
point $S$ to target point $M$ is $L$, and obstacles $O_{1}, O_{2}, \ldots, O_{K}$ are distributed between $S$ and $M$.

(1) Through parallel moving coordinate $O-X Y Z$ in the network $N(V, E)$ shown in Figure 3, point $O$ can coincide with $S$ point and the original point $O^{\prime}$ of the new coordinate can be obtained. In addition, the three original axes of $X Y Z$ are rotated by angle of $\varepsilon_{X}, \varepsilon_{Y}, \varepsilon_{Z}$, respectively, making $O^{\prime} Y^{\prime}$ axis coincide with $S M$ [29]. Thus the new coordinate system $O^{\prime}-X^{\prime} Y^{\prime} Z^{\prime}$ is obtained as shown in Figure 4.

The transformation relation between coordinate $O^{\prime}$ $X^{\prime} Y^{\prime} Z^{\prime}$ and $O-X Y Z$ is:

$$
\left[\begin{array}{l}
X \\
Y \\
Z
\end{array}\right]=\mathrm{R}\left[\begin{array}{c}
X^{\prime} \\
Y^{\prime} \\
Z^{\prime}
\end{array}\right]+\left[\begin{array}{c}
\Delta X \\
\Delta Y \\
\Delta Z
\end{array}\right]
$$

where $[\Delta X, \Delta Y, \Delta Z]^{\mathrm{T}}$ is translation matrix, Eq. (5) is rotation matrix $R$ :

$$
R=\left[\begin{array}{ccc}
\cos \varepsilon_{\mathrm{Y}} \cos \varepsilon_{\mathrm{Z}}-\sin \varepsilon_{\mathrm{Y}} \sin \varepsilon_{\mathrm{X}} \sin \varepsilon_{\mathrm{Z}} & -\cos \varepsilon_{\mathrm{Y}} \sin \varepsilon_{\mathrm{Z}}-\sin \varepsilon_{\mathrm{Y}} \sin \varepsilon_{\mathrm{X}} \cos \varepsilon_{\mathrm{Z}} & -\sin \varepsilon_{\mathrm{Y}} \cos \varepsilon_{\mathrm{X}} \\
\cos \varepsilon_{\mathrm{X}} \sin \varepsilon_{\mathrm{Z}} & \cos \varepsilon_{\mathrm{X}} \cos \varepsilon_{\mathrm{Z}} & -\sin \varepsilon_{\mathrm{X}} \\
\sin \varepsilon_{\mathrm{Y}} \cos \varepsilon_{\mathrm{Z}}+\cos \varepsilon_{\mathrm{Y}} \sin \varepsilon_{\mathrm{X}} \sin \varepsilon_{\mathrm{Z}} & -\sin \varepsilon_{\mathrm{Y}} \sin \varepsilon_{\mathrm{Z}}+\cos \varepsilon_{\mathrm{Y}} \sin \varepsilon_{\mathrm{X}} \cos \varepsilon_{\mathrm{Z}} & \cos \varepsilon_{\mathrm{Y}} \cos \varepsilon_{\mathrm{X}}
\end{array}\right] .
$$

The cube side $\mathrm{AE}=L$, and the coordinate of point $M$ is $(0, L, 0)$. The $O^{\prime} M$ is divided into $(n+1)$ equal segments. Through each equal diversion point, $n$ planes $\Pi_{i}(i=1,2$, $\ldots, n)$ which are parallel to ABCD can be obtained. The square plane $\Pi_{i}(i=1,2, \ldots, n)$ is divided into $m \times m$ equal little squares. Therefore, the real coordinate of the point $p(u, \omega, i) \in V(u, \omega=0,1, \ldots, m)$ on square plane $\Pi_{i}$ in coordination system $O^{\prime}-X^{\prime} Y^{\prime} Z^{\prime}$ is

$$
p^{\prime}\left(-\frac{L}{2}+\frac{u \cdot L}{m}, \frac{i \cdot L}{n},-\frac{L}{2}+\frac{\omega \cdot L}{m}\right) \in V^{\prime} .
$$

Step 2: Allow list calculation and goal conversion. Suppose there are totally $W=(w 1, w 2, \ldots)$ items of remanufacturing service information on network space $N^{\prime}\left(V^{\prime}\right.$, $E^{\prime}$ ) to be simultaneously transmitted from original point $\mathrm{S}(0,0,0)$.

Calculate the allowed $(u, i, \omega)(u, \omega=0,1, \ldots, m)$ of all points on plane $\Pi_{i}(i=1,2, \ldots, n-1)$ : suppose $p(u, \omega, i)$ is a certain point on plane $\Pi_{i}(i=1,2, \ldots, n-1)$, regarding
(2) In coordinate system $O^{\prime}-X^{\prime} Y^{\prime} Z^{\prime}$, the cube $A B C D$ EFGH is established, which constructs the information space network $N^{\prime}\left(V^{\prime}, E^{\prime}\right)$ shown in Figure 4. In the network, the ABCD surface of the cube, which is basically a square plane with side length of $L$, is on $X^{\prime} Z^{\prime}$ plane. Side $\mathrm{AB}$ coincides with $X^{\prime}$ axis, side $\mathrm{BC} / / Z^{\prime}$ axis, and the original point $O^{\prime}$ and point $A$ are in the same position.

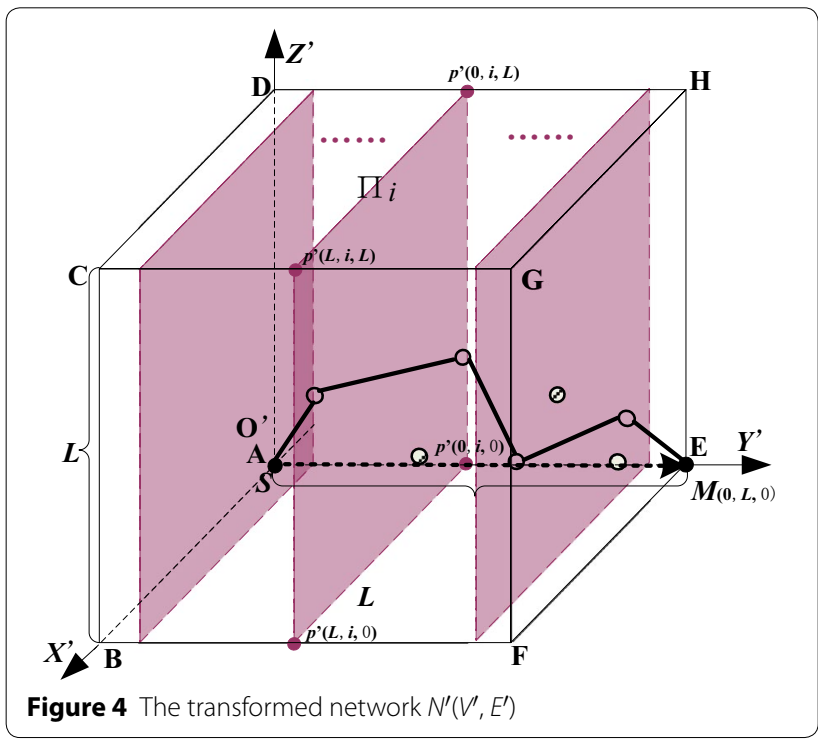

a random point $p(k, i+1, q)(k, q=0,1, \ldots, m)$ on plane $\Pi_{i+1}$, if $p(k, i+1, q)$ is fault-free, the information on line segment $p(u, i, \omega) p(k, i+1, q)$ can be successfully transmitted, therefore point $p(k, i+1, q)$ can be added into allowed $(u, i, \omega)$. Based on such method, all allowed reachable points of $p(u, i, \omega)$ can be calculated and stored in allowed $(u, i, \omega)$. Remove all nodes outside of allowed list as well as links that dissatisfy bandwidth constraints.

Suppose the properties of path form point $p(u, i, \omega)$ to point $p(k, i+1, q)$ is $<\operatorname{delay}\left(T\left(p_{i}, p_{i+1}\right)\right)$, $\operatorname{cost}\left(T\left(p_{i}, p_{i+1}\right)\right)$, packet_loss $\left(T\left(p_{i}, p_{i+1}\right)\right)>$, thus the problem of information transmission path optimization can be transformed into calculating the minimum of the weighted path properties, that is MIin $O\left(p_{i}, p_{i+1}\right)=\left[\operatorname{delay}\left(T\left(p_{i}, p_{i+1}\right)\right) \operatorname{cost}\left(T\left(p_{i}\right.\right.\right.$, $\left.\left.p_{i+1}\right)\right)$ packet_loss $\left.\left(T\left(p_{i}, p_{i+1}\right)\right)\right] W\left(p_{i}, p_{i+1}\right) . W\left(p_{i}, p_{i+1}\right)$ is weight matrix of the path properties.

Step 3: Initialization of the pheromone list. Initialize the pheromone list $\tau_{u \omega}^{i}$ of all points $p(u, i, \omega)(u$, $\omega=0,1, \ldots, m)$ on plane $\Pi_{i}(i=1,2, \ldots, n-1)$ : pheromone list is an array, where each data is used to represent the joint strength of pheromone between $p(u, i, \omega)$ and $p(k, i+1, q)$. Suppose the initialized pheromone $\tau_{u \omega}^{i}(0)=\mathrm{A} \quad \Delta \tau_{u \omega}^{i}(0)=0$ ( $\mathrm{A}$ is a certain constant).

Step 4: Calculation of transition probability. According to heuristic information value and pheromone value, in each procedure of information transmission path establishment in coordinate system $O^{\prime}-X^{\prime} Y^{\prime} Z^{\prime}$, 
the next node of $p(u, i, \omega)$ in information transmission is determined based on Eq. (6). $J$ is a random variable produced according to probability distribution shown in Eq. (7). $r$ is a constant within $[0,1]$, and $r_{0}$ is evendistributed random number within $[0,1]$.

$$
p_{i+1}=\left\{\begin{array}{cc}
\arg \max _{(k, i+1, q) \in \operatorname{allowed}(u, i, \omega)}\left[\tau_{i+1} \cdot \eta_{i, i+1}\right], & r<r_{0}, \\
J, & r>r_{0} .
\end{array}\right.
$$

Regarding the to-be-transmitted information of random point $p(u, i, \omega)$ on plane $\Pi_{i}(i=1,2, \ldots, n)$, the probability of $p(k, i+1, q)$ on plane $\Pi_{i+1}$ is selected, which is shown as Eq. (7):

$$
p_{i, i+1}=\left\{\begin{array}{cc}
\tau_{i+1} \cdot \eta_{i, i+1}^{\beta} & p(k, i+1, q) \in \text { allowed }, \\
\sum \tau_{i+1} \cdot \eta_{i, i+1}^{\beta} & p(k, i+1, q) \notin \text { allowed }, \\
0, & \text { ald }
\end{array}\right.
$$

where $\tau_{i+1}$ is the amount of pheromone stored on point $p(k, i+1, q), \eta_{i, i+1}=1 / d\left(p_{i}, p_{i+1}\right)$ is heuristic function, $\beta=\left\{\begin{array}{l}(4 t-2 r) / t, 0 \leq r \leq t \\ 2, t \leq q\end{array}\right.$ ( $t$ is the critical time) is heuristic value, $\beta$ reflects the level of emphasis that has been placed on total heuristic information in selection of information transmission paths during information transmission process.

Step 5: Select the next node and perform partial updating. At each node, to which information is transferred, Eq. (8) will be immediately used to perform real time updating of partial pheromone. If information arrives at certain node where no proper next node can be sought, such information is regarded invalid.

$$
\tau_{u \omega i}=(1-\mu) \tau_{u \omega i}+\mu \tau_{0}
$$

where $\tau_{u \omega i}$ is pheromone of the note $p(u, i, \omega), \mu$ is a certain parameter between $0-1, \tau_{0}$ is the initial value of pheromone of each feasible point.

Step 6: Judge whether arriving at target node or not. In judging whether the transmitted information meet with each other at certain point, if there exists information meeting, then meeting strategy operation will be performed and new path will be generated according to Eq. (9) and placed into path table. After that, the updating process is performed according to Eq. (10). Otherwise, transit to procedure (4).

$$
\begin{aligned}
& L_{\text {new }}=L\left(w_{1}\right)+L\left(w_{2}\right)\left(w_{1} \text { meets } w_{2}\right), \\
& \tau=(1-\mu) \cdot \tau+\mu \cdot \Delta \tau,
\end{aligned}
$$

where $L_{\text {new }}$ represents the length of the new path that is generated when path length $L\left(w_{1}\right)$ meets path length $L\left(w_{2}\right), \Delta \boldsymbol{\tau}=1 / L_{\text {new }}$.
Step 7: Global updating. Perform global pheromone updating according to Eq. (11):

$$
\tau_{u \omega i}=(1-\rho) \cdot \tau_{u \omega i}+\rho \cdot \Delta \tau_{u \omega i}
$$

where $\rho(0<\rho<1)$ is the pheromone volatility; $1-\rho$ is attenuation degree of pheromone with time; $\Delta \tau_{u \omega i}=\sum_{i=1}^{W} \Delta \tau_{u \omega i}^{w}$ is the information gain of each path after performing iteration $\left(\Delta \tau_{u \omega i}^{w}=1 / L_{\mathrm{w}}\right.$, the $w$ th information goes through the node $p(u, i, \omega)$ in current transmission process; otherwise $\Delta \tau_{u \omega i}^{w}=0$ ).

Step 8: Judge stop condition. Judge whether the algorithm meets the stop condition. If the stop condition is met, the optimized results can be output, otherwise turn to step 4. After all information to be transferred go through step 1 to step 8, the path from $S$ to $M$ is sought successfully.

\subsection{The Further Improved Ant-Genetic Algorithm (AC-GA) for Path Planning}

Since optimization method of 3D information transmission path based on improved AC in Section 5.1 is inferior for its large computation amount and over long time consuming, a further improved algorithm combing AC with GA (Genetic Algorithm) was proposed. The first step is to produce multiple paths produced by less amount of ants and collect network information by referring the ant distribution feature. The second step is to regard each successful path as an individual of GA and perform further optimization at sink node, so as to determine route table eventually. The operation flow of AC-GA-based 3D path optimization is shown in Figure 5.

According to the operation flow of AC-GA-based 3D path optimization, and based on initial population established by genetic algorithm and collected node

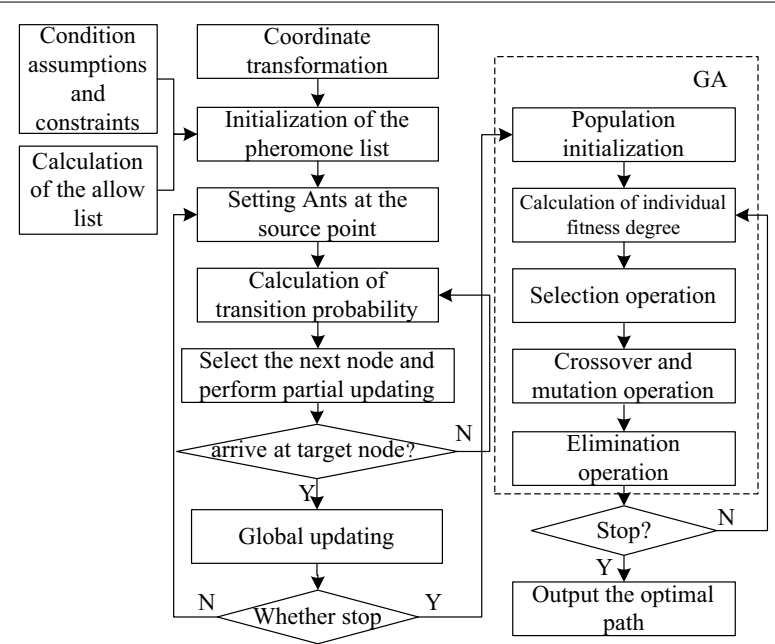

Figure 5 AC-GA-based 3D path optimization operation flow 
information, the optimized route can be determined, where the calculation steps are shown from step 9 to step 14.

Step 9: Population initialization. Each ant reaching sink node in stipulated cycle (an alternate path) is regarded as an individual of population $P(t)$. The individual coding is conducted by using ID number of node as gene code. When the stipulated population size is met, the AC algorithm is finished, otherwise turn to step 4.

Step 10: Calculation of individual fitness degree. Through population initialization, each individual fitness degree $F$ can be calculated by Eq. (12), and then $F$ is ranked in the order from big value to small value, $N$ individuals $(N<M)$ :

$$
F(x)=\sum_{i=1}^{n_{x}-1} E_{i+1}^{\alpha} R_{i, i+1}^{\beta} / n_{x}^{\lambda}
$$

where $E_{i+1}$ is the communication cost of node $i+1, i+1$ is the selected next node. $R_{i, i+1}$ is the signal strength received by node $i+1 . x$ is the path from original point to sink node; $n_{x}$ is the hop count of $x . \alpha, \beta, \lambda$ are weight constants. Such function is designed to balance routing efficiency and cost, thus realizing relatively higher costbenefit ratio.

Step 11: Selection operation. The population $P(t)$ was subjected to proportional selection as well as probability selection using Eq. (13). Therefore, some individuals with larger fitness degree are more likely to be preserved to the next generation.

$$
p_{1}=F / \sum_{i=1}^{M} F_{i}, i=1,2,3, \cdots, M .
$$

Step 12: Crossover and mutation operation. The individual was subjected to single-point crossover operation, where the crossover point will be selected between two individuals with the same gene. If both individuals share two pairs of the same genes, then either pair of gene can be selected, otherwise cancel the crossover operation.

Individual $i$ was subjected to mutation operation. Mutant gene was selected from collection $\Omega, \Omega=N_{\mathrm{b}}\{i\}$ $\{i-1\} \cap N_{\mathrm{b}}\{i\}\{i+1\} ; i-1$ and $i+1$ respectively represent both neighbours of node $i ; N_{\mathrm{b}}\{i\}$ represents the collection of nodes which are of effective communication. If $\Omega \in \Phi$, mutation operation can be avoided, otherwise evolutionary algorithm is performed for such individual to remove the same genes, and thus avoid being trapped into endless loop.

Step 13: Elimination operation. By mixing $M$ individuals with $N$ individuals, a new population including $M+N$ individuals in step 9 and step 12 can be obtained. After that the individuals with lower fitness degree were multiplied with penalty factor, which can be shown in Eq. (14):

$$
F(x)=\mu \cdot F_{\min }(x)
$$

where $\mu \in(0,1)$ is penalty factor, $F_{\min }(x)$ is the individuals with lower fitness degrees.

Step 14: Judge whether the algorithm is finished or not. The change rate $\xi$ of fitness degree is defined as:

$$
\xi=\sum_{i=n-j}^{n}\left|F_{i}(x)-\sum_{i=n-j}^{n} F_{i}(x) / j\right| / j
$$

where $\delta$ is a threshold value of change rate of fitness degree. $j$ is the number of iterations from $n$ to $n-j . n$ is the current number of iterations. When $\xi>\delta$, it turns to step 10 , otherwise the algorithm is finished.

\section{Numerical Study}

\subsection{Illustrative Example}

Taking the certain roller remanufacturing service demand information transmission of certain roller as example, suppose the coordinate of real-time routing node where user submits service demand is $S(3,1,9)$, while the coordinate of routing node where the platform accepts demand information of remanufacturing service is $M(16,16,6)$. The network for sending the information in remanufacturing service system is $100 \mathrm{Mbit} /$ Ethernet.

The experimental simulation constraint condition is: after coordinate transformation, the $S^{\prime} M^{\prime}$ in the $Y^{\prime}$ direction was divided into 16 equal segments. After non-dimensionalization, the $2 \mathrm{D}$ plane was divided into $16 \times 16$, each grid's length was set to 1 . The designed 3D path planning AC-GA algorithm was programmed using MATLAB R2012a. The optimized communication path from original point $S(3,1,9)$ to $M(16,16,6)$ was simulated, resulting in the optimized 3D path simulation results in $O^{\prime}-X^{\prime} Y^{\prime} Z^{\prime}$ (Figure 6(a)) and in $O-X Y Z$ (Figure 6(b)) and path coordinate table (Table 2). According to Eqs. (1) and (2), along such optimized path, the information transmission delay is $206.5148 \mu$ s and the communication cost is 20.0588. According to Eq. (3), information packet_loss is $7.7375 \%$ while the average information load factor is $53.4723 \%$. Finally, the roller remanufacturing service demand information was transferred along the optimized path specified in Table 2.

\subsection{Performance Results and Discussion 6.2.1 Result Validation}

Through calculating the optimal fitness degree of AC-GA algorithm, it can further verify the superiority of the optimized 3D path, as shown in Table 2. According to Eq. (12), the changing process of cost effectiveness fitness of AC-GA algorithm during the process of 3D path 


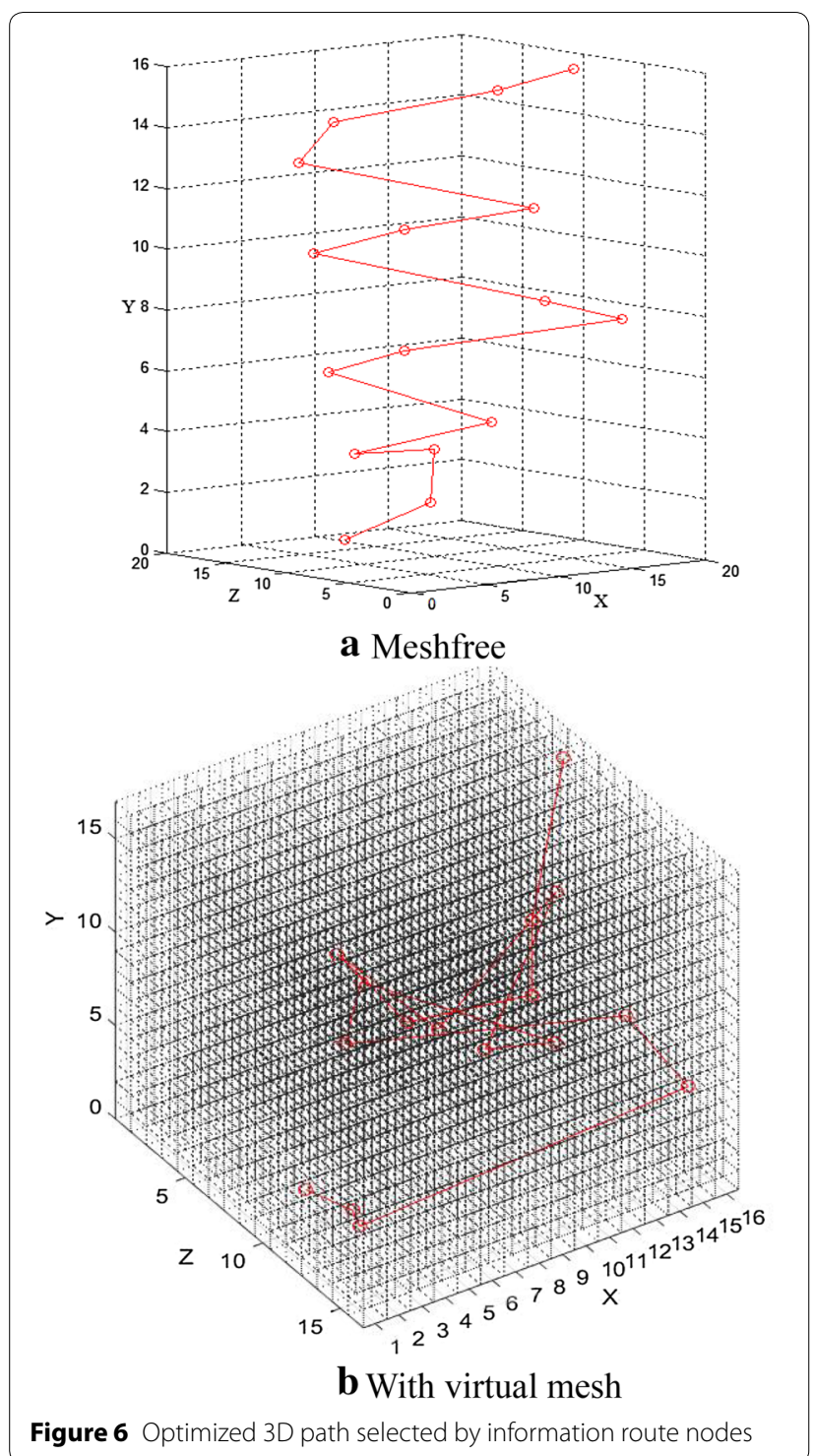

Table 2 Node coordination of optimized 3D path

\begin{tabular}{llll}
\hline$(3,1,9)$ & $(8,2,8)$ & $(11,3,13)$ & $(2,4,7)$ \\
$(16,5,10)$ & $(6,6,7)$ & $(13,7,14)$ & $(9,8,12)$ \\
$(10,9,1)$ & $(3,10,11)$ & $(4,11,11)$ & $(10,12,2)$ \\
$(4,13,14)$ & $(8,14,16)$ & $(6,15,1)$ & $(16,16,6)$ \\
\hline
\end{tabular}

optimization can be obtained, shown in Figure 7. The initial cost effectiveness fitness was 101.2841, which was then processed by 17 iterations, reaching a steady value of 86.292. As shown in Figure 7 it can be seen that the initial fitness degree of AC-GA algorithm is relatively large and the alternative paths of route information nodes are in a considerable quantity, which should be further screened.
With increased number of iterations of $\mathrm{AC}$ algorithm, the fitness degree of GA algorithm changes accordingly, which means the number of alternative paths is gradually decreased. When the fitness degree tends to be steady, i.e., the number of alternative path is 0 , the optimized path in Table 2 can be sought.

\subsubsection{Performance Analysis of AC-GA Algorithm}

Through comparative analysis between improved AC in Section 5.1 and AC-GA in Section 5.2 in terms of $\operatorname{delay}(T(S, M))$, $\operatorname{cost}(T(S, M))$, packet_loss $(T(S, M))$ during the optimization process of information transmission path planning, the performance analysis of AC-GA algorithm can be realized.

\section{(1) Analysis of information transmission delay}

Suppose the unquantified distances between the adjacent nodes in the information transmission path are $1 \mathrm{~km}, 5 \mathrm{~km}, 10 \mathrm{~km}, 15 \mathrm{~km}$ and $20 \mathrm{~km}$. According to Eq. (1), the information transmission delays $(T(S, M))$ of improved AC and AC-GA algorithm during the optimization process of information transmission path planning can be calculated, respectively. The comparison results are shown in Figure 8.

As shown in Figure 8, when the transmission distance is relatively short, there is no significant difference in delay between the improved AC and AC-GA algorithm; however with the increase of transmission distance, AC-GA algorithm gradually shows its significant advantages, which indicates that the longer the transmission distance is, the more superior the transmission path established by AC-GA algorithm is.

(2) Analysis of information communication cost $(T(S$, $M)$ )

According to Eq. (2), the information communication costs $(T(S, M))$ of improved AC and AC-GA algorithm under 3 different meshing situations of $9 \times 9,16 \times 16$, $23 \times 23$, can be calculated respectively. The comparison results of both total communication costs under 3 different meshing situations are shown in Figure 9. It can be seen that with the increase of network scale, the communication cost of AC-GA algorithm is significantly lower than that of improved AC.

\section{(3) Analysis of information packet_loss $(T(S, M))$}

According to Eq. (3), the information packet_loss $(T(S$, $M)$ ) of two algorithms when network node average load factor is $10 \%, 20 \%, 30 \%, 40 \%, 50 \%, 60 \%, 70 \%, 80 \%, 90 \%$ can be calculated, respectively, and the results are shown 


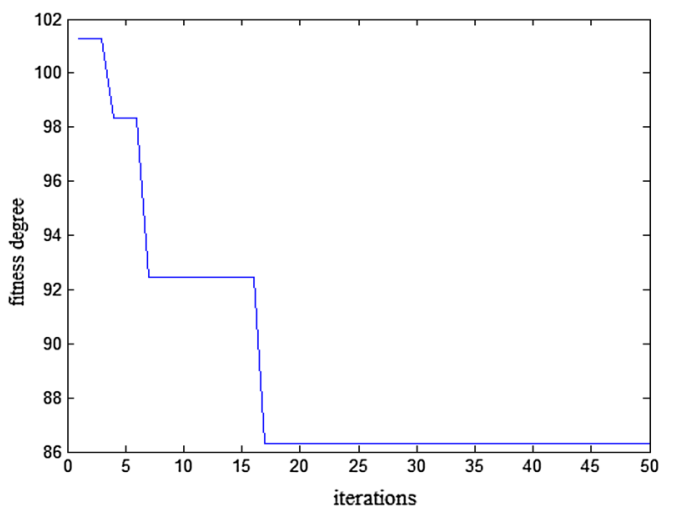

Figure 7 Optimization process of cost effectiveness fitness in AC-GA algorithm

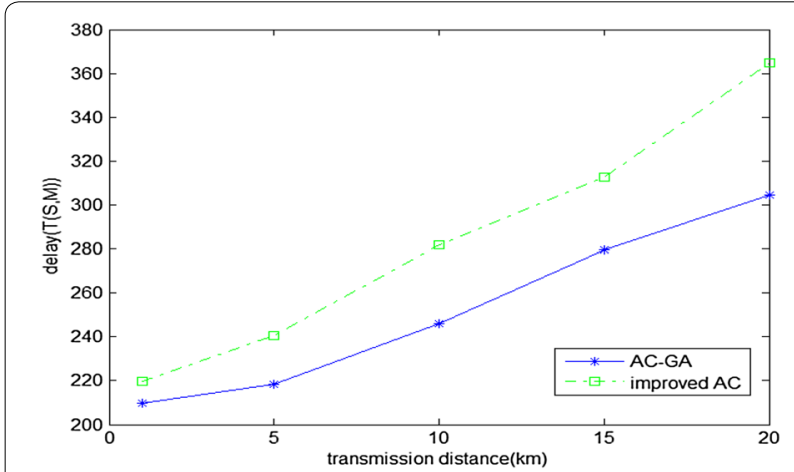

Figure 8 Comparison of information transmission delays $(T(S, M))$ between improved $A C$ and $A C-G A$ algorithm in Figure 10. It can be seen that the higher the information load factor is, the higher the information packet $\operatorname{loss}(T(S, M))$ will be.

\section{Conclusions}

The 3D information transmission path optimization for remanufacturing service system network framework plays a vital role in quickly and accurately selecting the optimal information transmission path as well as in decreasing information transmission delay and information packet_loss.

(1) By referring the service-oriented manufacturing thought and combining the features of remanufacturing and service, the concept of remanufacturing service was proposed and a 3D framework for remanufacturing service information transmission network was built.

(2) Since the traditional ant colony algorithm (AC) is only suitable for two-dimensional path planning but not applicable for three-dimensional path planning, an improved 3D ant colony algorithm (Improved $\mathrm{AC}$ ) was proposed to solve the problem of $3 \mathrm{D}$ path optimization in the information network.

(3) To further improve the convergence of the algorithm and decrease the number of iterations, this paper proposed an improved ant colony-genetic algorithm (AC-GA) by combining ant colony algorithm and genetic algorithm to realize the 3D infor-

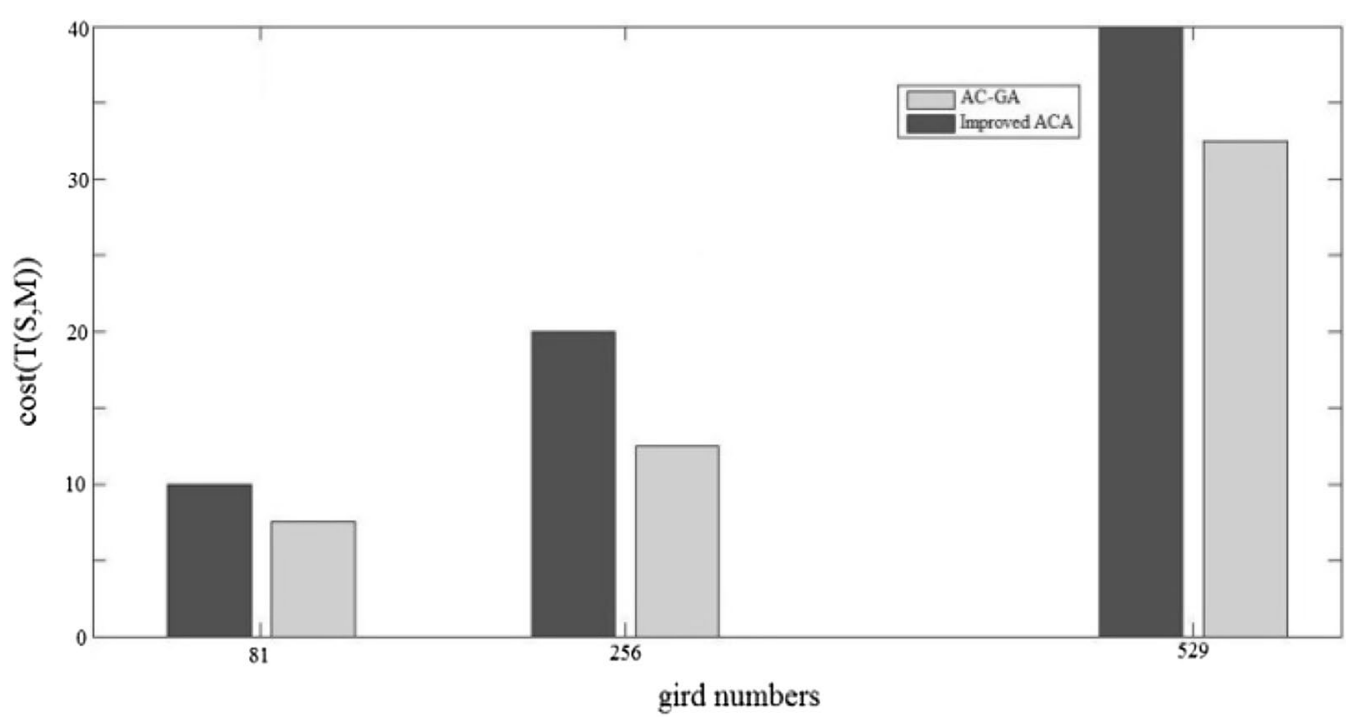

Figure 9 Comparison of communication costs $(T(S, M))$ of improved $A C$ and $A C-G A$ algorithm 


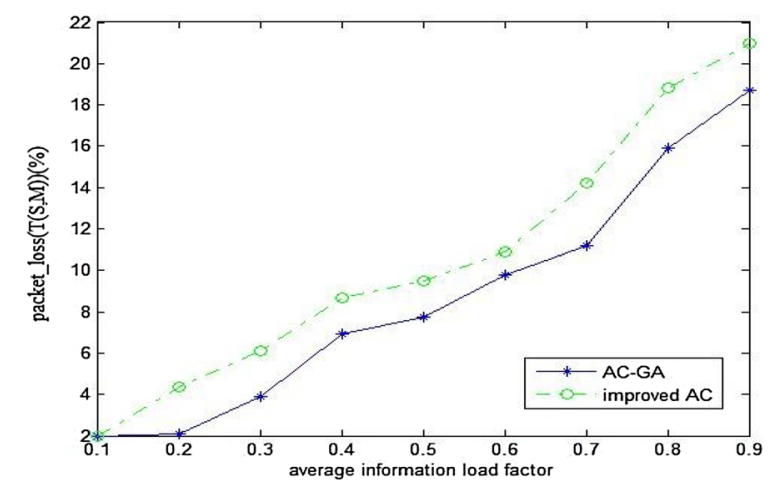

Figure 10 Comparison of packet_loss $(T(S, M))$ of improved $A C$ and AC-GA algorithm

mation transmission path optimization in remanufacturing service system.

(4) Taking the remanufacturing service demand information transmission of certain roller as example, the effectiveness of such algorithm was analyzed. Through comparative analysis of performances, it can be demonstrated that the AC-GA algorithm has good operation efficiency in information transmission path optimization. In addition, AC-GA algorithm has advantages over improved $\mathrm{AC}$ in aspects of information transmission delay, information communication cost and information pakect_loss. This study lays foundation for remanufacturing service system research, and provides the basic theories on the system information transmission as well as references for the research and application of remanufacturing service in other industries.

\section{Authors' Contributions}

$\mathrm{X}-\mathrm{HX}$ was in charge of the whole trial; LW wrote the manuscript; J-HC, XL and J-WL assisted with sampling and laboratory analyses. All authors read and approved the final manuscript.

\section{Author Details}

1 Hubei Key Laboratory of Mechanical Transmission and Manufacturing Engineering, Wuhan University of Science and Technology, Wuhan 430081, China.

${ }^{2}$ Center for Service Science and Engineering, Wuhan University of Science and Technology, Wuhan 430065, China.

\section{Authors' Information}

Lei Wang, born in 1987, is currently a lecturer at Hubei Key Laboratory of Mechanical Transmission and Manufacturing Engineering, Wuhan University of Science and Technology, China. Her main research interests include manufacturing/remanufacturing systems engineering, reverse supply chain management, knowledge management and engineering.

Xu-Hui Xia, born in 1966, is currently a professor and PhD candidate supervisor at Wuhan University of Science and Technology, China. His main research interests include manufacturing systems engineering, supply chain management, optimization and decision with application in manufacturing industry.

Jian-Hua Cao, born in 1989, is currently an assistant engineer and PhD candidate at Wuhan University of Science \& Technology, China. His main research interests include manufacturing/remanufacturing systems engineering and reverse supply chain management.

Xiang Liu, born in 1983, is currently an engineer at Wuhan University of Science and Technology, China. His main research interests include image processing, computer vision and its application in manufacturing engineering.

Jun-Wei Liu, born in 1983, is currently a lecturer at Wuhan University of Science and Technology, China. His research interests are in the areas of manufacturing informatization and intelligent manufacturing.

\section{Competing Interests}

The authors declare that they have no competing interests.

\section{Funding}

Supported by National Natural Science Foundation of China (Grant Nos. 51805385, 71471143), Hubei Provincial Natural Science Foundation of China (Grant No. 2018CFB265), and Center for Service Science and Engineering of Wuhan University of Science and Technology (Grant No. CSSE2017KA04)

\section{Publisher's Note}

Springer Nature remains neutral with regard to jurisdictional claims in published maps and institutional affiliations.

Received: 3 May 2017 Accepted: 13 December 2018

Published online: 24 December 2018

\section{References}

[1] L Wang, X H Xia, Y Q Xiong, et al. Architecture of reverse supply chain service system. Computer Integrated Manufacturing Systems, 2015, 21 (10): 2720-2731. (in Chinese)

[2] RT Lund. Remanufacturing: The experience of the United States and implications for developing countries. Technology Review, 1984, 87(2): 18-23.

[3] P Goodall, E Rosamond, J Harding. A review of the state of the art in tools and techniques used to evaluate remanufacturing feasibility. Journal of Cleaner Production, 2014(81): 1-15.

[4] B Su, X M Huang, Y H Ren, et al. Research on selective assembly method optimization for construction machinery remanufacturing based on ant colony algorithm. Journal of Mechanical Engineering, 2017, 53(5): 60-68. (in (hinese)

[5] M Mitsutaka, K Shingo. Demand forecasting for production planning in remanufacturing. International Journal of Advanced Manufacturing Technology, 2015(79): 161-175.

[6] M Z Liu, X Zhang, C H Liu, et al. Optimization method of remanufacturing reprocessing shop scheduling under uncertain conditions. Journal of Mechanical Engineering, 2014, 50 (10): 206-212. (in Chinese)

[7] Y J He. Acquisition pricing and remanufacturing decisions in a closedloop supply chain. International Journal of Production Economics, 2015(163): 48-60

[8] L Wang, X H Xia, Y Q Xiong, et al. Modular method of remanufacturing service resources. Computer Integrated Manufacturing Systems, 2016, 22(9): 2204-2216. (in Chinese)

[9] W J Xu, S S Tian, Q Liu, et al. An improved discrete bees algorithm for correlation-aware service aggregation optimization in cloud manufacturing. International Journal of Advanced Manufacturing Technology, 2016(84): 17-28.

[10] D Lottridge, M Chignell, S E Straus. Requirements analysis for customization using subgroup differences and large sample user testing: A case study of information retrieval on handheld devices in healthcare. International Journal of Industrial Ergonomics, 2011, 41(3): 208-218.

[11] S Liaskos, S M Khan, M Litoiu., et al. Behavioral adaptation of information systems through goal models. Information Systems, 2012, 37(8): 767-783.

[12] G C S Lin, F F Yang, F Z Y Hu. The new geography of information and consulting services in China: Comparing Beijing and Guangzhou. Habitat International, 2012, 36(4): 481-492. 
[13] S H Ma, L Tian. A web service-based multi-disciplinary collaborative simulation platform for complicated product development. International Journal of Advanced Manufacturing Technology, 2014(73): 1033-1047.

[14] X Gao, Y W Fang, Y L Wu. Fuzzy Q learning algorithm for dual-aircraft path planning to cooperatively detect targets by passive radars. Journal of Systems Engineering and Electronics, 2013, 24(5): 800-810.

[15] S M Mohammad, D A Saeed, E Farshid, et al. An ant colony algorithm (ACA) for solving the new integrated model of job shop scheduling and conflict-free routing of AGVs. Computers \& Industrial Engineering, 2015, 86: 2-13.

[16] Y Shi, B Liang, X Q Wang, et al. Cartesian non-holonomic path planning of space robot based on quantum-behaved particle swarm optimization algorithm. Journal of Mechanical Engineering, 2011, 47(23): 65-73. (in Chinese)

[17] Y C Zhou, Y F Dong, H M Xia, et al. Routing optimization of intelligent vehicle in automated warehouse. Discrete Dynamics in Nature and Society, 2014, 2014(1): 1-14.

[18] J Berger, N Lo. An innovative multi-agent search-and-rescue path planning approach. Computers \& Operations Research, 2015(53): 24-31.

[19] B C Zhang, W Q Liu, Z L Mao, et al. Cooperative and geometric learning algorithm (CGLA) for path planning of UAVs with limited information. Automatica, 2014, 50(3): 809-820.

[20] H Hu, X S Cai. Improved ant colony algorithm-based three-dimensional robot path planning. Computer Systems \& Applications, 2011, 20(11): 95-98. (in Chinese)

[21] Z Q Wang, X G Zhu, Q Y Han. Mobile robot path planning based on parameter optimization ant colony algorithm. Procedia Engineering, 2011(15): 2738-2741.
[22] L R Li, L Wang, Y B Gao, et al. A dynamic path planning model based on the optimal ant colony algorithm. Journal of Guangxi University: Nat. Sci. Ed., 2013, 38(2): 359-367. (in Chinese)

[23] M N Li. Efficiency improvement of ant colony optimization in solving the moderate LTSP. Journal of Systems Engineering and Electronics, 2015, 26(6): 1301-1309.

[24] F Y Chen, HW Wang, C Qi, et al. An ant colony optimization routing algorithm for two order pickers with congestion consideration. Computers \& Industrial Engineering, 2013, 66(1): 77-85.

[25] F Yu, W H Liao, Y N Xie, et al. Application of continuous space ant colony algorithm to proliferative process route optimization. Journal of Computer-Aided Design \& Computer Graphics, 2013, 20(7): 952-956. (in Chinese)

[26] P P Maglio, S L Vargo, N Caswell, et al. The service system is the basic abstraction of service science. Information Systems and e-Business Management, 2009, 7(4): 395-406.

[27] G Yu, C J Zhong, XY Lan, et al. Research of multi-path routing based on network coding in space information networks. Chinese Journal of Aeronautics, 2014, 27(3): 663-669.

[28] Z S Zhong. Ant colony algorithm based on path planning for mobile agent migration. Procedia Engineering, 2011(23): 1-8.

[29] J W Liu, JY Kong, M Zhou, et al. An intelligent communication path planning method of metallurgical equipment multi-dimensional information space. Journal of Digital Information Management, 2012, 10(5): 295-300.

\section{Submit your manuscript to a SpringerOpen ${ }^{\odot}$ journal and benefit from:}

- Convenient online submission

- Rigorous peer review

- Open access: articles freely available online

- High visibility within the field

- Retaining the copyright to your article

Submit your next manuscript at $\boldsymbol{\nabla}$ springeropen.com 